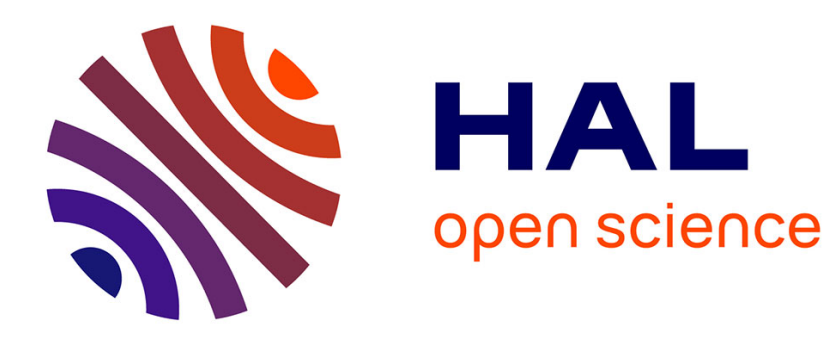

\title{
Les institutions sans but lucratif hier et aujourd'hui : comparaison France-Etats-Unis
}

Edith Archambault

\section{To cite this version:}

Edith Archambault. Les institutions sans but lucratif hier et aujourd'hui : comparaison France-EtatsUnis. The Tocqueville Review/La revue Tocqueville, 2011, XXXII (2), pp.81-98. halshs-00660859

\section{HAL Id: halshs-00660859 \\ https://shs.hal.science/halshs-00660859}

Submitted on 17 Jan 2012

HAL is a multi-disciplinary open access archive for the deposit and dissemination of scientific research documents, whether they are published or not. The documents may come from teaching and research institutions in France or abroad, or from public or private research centers.
L'archive ouverte pluridisciplinaire HAL, est destinée au dépôt et à la diffusion de documents scientifiques de niveau recherche, publiés ou non, émanant des établissements d'enseignement et de recherche français ou étrangers, des laboratoires publics ou privés. 


\title{
LES INSTITUTIONS SANS BUT LUCRATIF HIER ET AUJOURD'HUI : COMPARAISON FRANCE-ÉTATS-UNIS
}

\author{
Edith ARCHAMBAULT
}

« Partout où, à la tête d'une entreprise nouvelle, vous voyez en France le gouvernement et en Angleterre un grand seigneur, comptez que vous apercevez aux États-Unis une association", disait Tocqueville en 1840. Il est toujours intéressant 170 ans plus tard de comparer les Américains et les Français dans leur rapport à l'Etat et à l'initiative privée associative ${ }^{1}$. Certes, une véritable démocratie s'est instaurée en France depuis la Monarchie de Juillet. Les associations cependant, si intimement mêlées à la démocratie selon Tocqueville, ont été longues à se multiplier et à prendre de l'ampleur car elles ont été contrôlées et réprimées par les régimes autoritaires qui ont périodiquement mis entre parenthèses la République. C'est seulement après la Seconde Guerre mondiale que la liberté d'association, tardivement légalisée en 1901, a été retrouvée définitivement en France 2 . Après 1960-1970, les associations se multiplient rapidement et le secteur sans but lucratif français rattrape son retard par rapport à celui des démocraties développées.

Dans la comparaison socio-économique qui va suivre, on retiendra un terme plus neutre, moins juridique et donc moins institutionnellement connoté que celui d'associations, celui d'institutions sans but lucratif (ISBL). C'est le terme statistique utilisé par le Système international de comptabilité nationale (SCN93) qui en donne définition suivante :

«Les institutions sans but lucratif sont des entités juridiques ou sociales créées dans le but de produire des biens ou des services, dont le statut ne leur permet pas d'être une source de revenu, de profit ou 
d'autres formes de gain financier, pour les unités qui les créent, les contrôlent ou les financent $»^{3}$.

Cette définition est précisée et rendue opérationnelle par le Manuel sur les Institutions sans but lucratif dans le Système de Comptabilité nationale de l'ONU (ONU, 2006). Le secteur sans but lucratif regroupe alors «l'ensemble des unités qui sont simultanément:

- organisées, ni éphémères, ni spontanées ;

- sans but lucratif, c'est-à-dire ne distribuant pas le profit qu'elles peuvent éventuellement réaliser ;

- privées, institutionnellement distinctes des administrations publiques ;

- auto-administrées, sans contrôle d'une autre entité ;

- à participation volontaire, ni automatique, ni obligatoire ${ }^{4} »$.

Concrètement, le concept d'ISBL recouvre en France essentiellement les associations et les fondations, à condition qu'elles remplissent simultanément les cinq critères précédemment énoncés ${ }^{5}$, alors qu'aux Etats-Unis, c'est l'Internal Revenue Service, l'autorité fiscale, qui détermine, sur documents au moment de leur enregistrement, quelles entités sont non lucratives et donc exemptées des impôts sur les sociétés. Le secteur sans but lucratif américain ainsi déterminé est composé de public charities et de private foundations. Les private charities ont un objet "charitable ", c'est-à-dire d'intérêt général: il porte limitativement sur la religion, les arts, la santé, l'éducation, la recherche, les services humains. Les private foundations ont les mêmes objectifs le plus souvent, mais ce n'est pas limitatif; elles tirent une part essentielle de leurs ressources des revenus d'une dotation initiale (endowment). A ces deux catégories qui œuvrent pour le bien public s'ajoutent des organisations qui travaillent pour les intérêts de leurs membres : syndicats, organisations professionnelles, Health management organizations, groupes de défense des droits et des intérêts, clubs récréatifs ${ }^{6}$.

La comparaison des secteurs sans but lucratif (SSBL ou Tiers secteurs) américain et français commencera par un état des lieux en 1995, tel qu'il ressort de la deuxième phase du Programme Johns Hopkins?. Les données empiriques strictement comparables sont interprétées grâce à des ideal-types, car les tiers secteurs des EtatsUnis et de la France sont considérés comme représentatifs respectivement du modèle libéral anglo-saxon et du modèle 
partenarial européen de bien-être (Salamon et Anheier, 1998). Dans un deuxième temps, l'évolution des ISBL dans les deux pays depuis ce point de départ jusqu'à 2007 sera analysée, en insistant sur le renforcement des spécificités de chaque pays, sur leur forte « dépendance de sentier» (Nelson et Winter, 1983), même si certaines tendances se retrouvent dans les deux pays. Enfin on essaiera de voir l'impact de la crise financière, économique et sociale sur les organisations non lucratives. Bien sûr, les données empiriques ne sont pas disponibles actuellement et on s'appuiera sur des enquêtes plus qualitatives et des dires d'expert. On ne peut donc qu'émettre des hypothèses sur la résilience respective des deux Tiers secteurs.

I - LES TIERS SECTEURS AMERICAIN ET FRANÇAIS A LA FIN DU XX ${ }^{\mathrm{E}}$ SIECLE : MODELE LIBERAL VERSUS MODELE PARTENARIAL ?

Le Programme de comparaison internationale du secteur sans but lucratif avait, entre autres buts, celui de combler l'invisibilité de ce secteur dans les comptes nationaux. La seconde phase de ce programme a mobilisé 37 pays et portait sur l'année $1995^{8}$. Ce sont ces données comparables qui vont permettre de présenter le poids économique, la composition par domaine d'activité et l'origine des ressources des deux tiers secteurs à la fin du $\mathrm{XX}^{\mathrm{e}}$ siècle (a). Puis on interprétera ces données économiques à travers la grille de lecture de la théorie des origines sociales (Salamon and Anheier, 1998) que nous avons précisée pour l'Europe au cours de travaux antérieurs (Archambault, 2009) (b)

$a$ - Le Tiers secteur en France et aux Etats-Unis en 1995 : que disent les chiffres?

On voit dans le tableau 1 le poids économique de l'ensemble des ISBL telles qu'elles ont été précédemment définies. Cependant, seules celles qui emploient des salariés sont en fait prises en compte, faute de sources statistiques sur celles qui ne vivent que grâce à l'engagement bénévole. Les cultes et congrégations religieuses par ailleurs ont été omis, car ils sont impossibles à cerner dans la plupart des pays. Cependant la mesure du bénévolat permet de pallier la première lacune et on verra ultérieurement l'importance inégale de la variable religieuse pour les deux pays. 
Tableau 1 - Poids économique du SSBL en France et aux Etats-Unis, 1995

\begin{tabular}{|l|c|c|}
\hline & France & Etats-Unis \\
\hline Salariés en équivalent temps plein (ETP) & 960000 & 8555000 \\
\hline Salariés/emploi total ETP & $4,9 \%$ & $7,8 \%$ \\
\hline Bénévoles ETP & $630000^{9}$ & 4995000 \\
\hline Dépenses courantes (M\$) & 57000 & 502000 \\
\hline
\end{tabular}

Source : Salamon et associates, 1999.

On voit donc qu'en valeur absolue le tiers secteur américain, le plus grand du monde, est presque dix fois plus important que son homologue français, ou, si l'on tient compte des différences de population, environ le double. En valeur relative, l'écart se rétrécit un peu, car le taux d'emploi est plus élevé aux Etats-Unis qu'en France, mais il reste substantiel. Dans les deux pays, le SSBL est un employeur majeur, avec une main-d'œuvre très majoritairement féminine comme dans la plupart des activités de service. Seules les dépenses courantes de ce secteur ont été appréhendées faute d'information fiable sur les dépenses en capital, ce qui sous- estime un peu le poids économique du SSBL.

La composition interne des deux secteurs ${ }^{10}$ selon une nomenclature adaptée aux services rendus par les ISBL, International Classification of NonProfit Organizations (ICNPO), montre que les domaines d'activité relevant de la protection sociale dans une conception européenne, la santé et les services sociaux, polarisent l'essentiel de l'activité économique du tiers secteur dans les deux pays. Mais, alors qu'aux Etats-Unis c'est la santé qui représente prés de la moitié du secteur, en France la polarisation se fait autour de l'action sociale, avec $40 \%$ de l'emploi salarié. On commentera ultérieurement cette différence fondamentale qui relève d'une attitude différente des deux peuples à l'égard de l'Etat. Dans les deux pays, le poste "éducation et recherche » arrive en second et représente un cinquième du SSBL, mais il s'agit de l'enseignement primaire et secondaire privé sous contrat en France, catholique à 95\%, alors qu'aux Etats-Unis ce sont surtout les universités privées, des plus prestigieuses de l'Ivy League - Harvard, Princeton, Yale, Columbia - à celles qui sont moins connues. Dans le domaine de la culture, la plupart des musées et des orchestres sont des ISBL aux Etats-Unis alors que les associations sportives, culturelles et de loisir sont petites et très nombreuses en France et fonctionnent essentiellement avec 
des bénévoles. Le développement local ou community development, comme les associations civiques et de défense des droits, ont le même poids et le même rôle dans les deux pays, alors que les organisations professionnelles sont relativement plus importantes outre-Atlantique. Enfin, les associations écologiques ou de solidarité internationale ont un poids économique quasi-nul aux Etats-Unis et on remarque l'absence de fondations collectant et redistribuant des fonds en France en 1995, à l'exception de la Fondation de France.

Comment les ISBL se financent-elles dans chaque pays ? Elles recourent très différemment au financement public et au financement privé. Les ressources d'origine publique mêlent les subventions sans contrepartie directe et les contrats, remboursements ou prix de journée qui sont considérés comme des prestations de services ou des ventes au secteur public. Les ressources privées sont les participations des bénéficiaires au service rendu (généralement inférieures au coût de production et souvent modulées en fonction du revenu) les ventes accessoires, les revenus de placements qui concernent principalement les fondations. A côté de ces ressources propres, certaines ISBL, plus nombreuses aux Etats-Unis qu'en France, reçoivent des dons et des legs des particuliers et diverses formes de mécénat d'entreprise. Cependant dans les deux pays ces ressources philanthropiques n'ont qu'un rôle d'appoint pour l'ensemble du tiers secteur et elles sont concentrées sur un petit nombre d'ISBL $(7 \%$ des ressources du secteur en France, 13\% aux Etats-Unis).

La majorité des sources de financement du Tiers secteur français est publique (58\%), en provenance de l'Etat, de la Sécurité sociale et des collectivités territoriales, alors que les ressources commerciales, et notamment les droits payés par les usagers (fees), prédominent clairement aux Etats-Unis, avec 57\% des ressources. Cependant le financement public, contrairement aux idées reçues, y est loin d'être négligeable et représente $30 \%$ des ressources, alors que les ressources propres en France s'élèvent à un peu plus du tiers du budget du SSBL.

Ces sources de financement, comme les différences de composition et de rôle des tiers secteurs indiquent évidemment un rapport de la société civile à l'Etat et au marché différent dans les deux pays. 
$b$ - Les Tiers secteurs américain et français confrontés à la grille de lecture de la théorie des origines sociales

La théorie des origines sociales (Salamon et Anheier, 1999) explique les différences entre Tiers secteurs par leurs racines historiques profondes dans tous les pays, par les rapports de force entre les diverses classes sociales et par la relation qui prévaut dans chaque pays entre Etat et société. Par ailleurs, les ISBL ont été un enjeu dans les conflits historiques entre Eglise et Etat qui déterminent encore largement leurs rapports actuels avec ces institutions.

Le tableau 2 schématise quatre idéal-types de relations entre ISBL et Etat social, en croisant deux critères : la taille relative du tiers secteur et le niveau relatif de la dépense sociale financée par les pouvoirs publics :

Tablean 2 : Les quatre ideal-types de SSBL, selon la théorie des origines sociales

\begin{tabular}{|l|c|c|}
\hline $\begin{array}{c}\text { Dimension du SSBL } \\
\text { Dépense sociale publique }\end{array}$ & Basse & Haute \\
\hline Basse & $\begin{array}{c}\text { Etatiste } \\
\text { (Japon ; pays en } \\
\text { développement) }\end{array}$ & $\begin{array}{c}\text { Libéral } \\
\text { (pays anglo- } \\
\text { saxons) }\end{array}$ \\
\hline Haute & $\begin{array}{c}\text { Social-démocrate } \\
\text { (pays scandinaves) }\end{array}$ & $\begin{array}{c}\text { Partenarial ou } \\
\text { corporatiste } \\
\text { (Europe } \\
\text { continentale) }\end{array}$ \\
\hline
\end{tabular}

Source : Salamon et Anheier, 1998.

Cette typologie des Tiers secteurs est évidemment à rapprocher de celle des Etats de bien-être d'Esping Andersen (1990). Etat social et Tiers secteur ont des rapports de substitution dans les modèles libéral et social-démocrate, alors qu'ils sont en complémentarité dans les modèles étatiste et partenarial. Le SSBL américain peut être considéré comme un exemple du modèle libéral et son homologue français comme représentatif du modèle corporatiste.

Dans les deux pays les institutions sans but lucratif ont précédé historiquement l'Etat social. Un système de protection sociale n'est apparu en France que dans les années 1930 et surtout après 1945 avec l'extension progressive de la Sécurité sociale. Aux Etats-Unis, il faudra attendre 1965 et la Great Society du Président Johnson pour voir l'Etat instaurer un régime de protection, mais beaucoup plus partiel qu'en France, contre la maladie et la vieillesse. Intimement mêlées à la 
démocratie américaine dès ses origines, les associations, souvent liées à des dénominations religieuses concurrentes, ont pallié l'absence d'Etat ou ses carences par la pratique du «self help» lors de la conquête de l'Ouest. Cette tradition perdure puisque les ISBL y remplissent actuellement des fonctions qui relèvent en France principalement du secteur public : plus de la moitié des hôpitaux sont privés et non lucratifs, de même que les universités les plus prestigieuses. Alors qu'en France les ISBL travaillent plutôt en partenariat avec les pouvoirs publics, en fonction du principe de subsidiaritée ${ }^{11}$, aux Etats-Unis elles sont en concurrence avec les pouvoirs publics, notamment dans le domaine de l'enseignement supérieur, et avec le secteur lucratif dans celui de la santé. Corrélativement, le financement public est la ressource prédominante du SSBL en France comme dans la plupart des pays européens, alors que les ventes de services prédominent aux Etats-Unis.

Le poids et la nature du système de protection sociale façonnent fortement les interrelations entre Etat et SSBL: principe de la responsabilité individuelle et assistance limitée sous condition de ressources et de règles de droit, dans le modèle libéral ; protection sociale très large, à base professionnelle préservant les statuts respectifs des classes sociales, dans le modèle corporatiste. L'opposition des systèmes juridiques, issu de la Common law aux EtatsUnis et héritier du droit romain en Europe continentale, explique aussi la présomption de non-lucrativité de quelques catégories juridiques dont évidemment les associations en France, alors que les organisations américaines, qui sont des sociétés commerciales ou corporations, ont toujours à prouver qu'elles ne distribuent pas de bénéfices.

Ces relations différentes s'expliquent par l'enracinement historique du SSBL dans les deux pays. Histoire brève aux Etats-Unis où les pionniers ont fait face à leurs besoins collectifs par l'association communautaire, avec une réticence durable à l'égard des ingérences de l'Etat fédéral. Histoire longue en France où le SSBL remonte aux œuvres charitables et aux corporations et confréries du Moyen-âge. En outre, au XIX ${ }^{\text {e }}$ siècle, la naissance des formes modernes de mutuelles et d'associations a été fortement liée en France au mouvement ouvrier alors que le SSBL américain est totalement indépendant du mouvement syndical. 
Cette histoire longue se traduit pour les associations en France par des références idéologiques multiples : socialisme utopique, christianisme social, marxisme populaire ou trotskiste, solidarisme, et même libéralisme, sans équivalents outre-Atlantique. Aux Etats-Unis, la référence à la tradition individualiste puritaine reste très prégnante dans de nombreuses organisations sans but lucratif, parce qu'elles sont nées dans un climat de concurrence entre dénominations religieuses, même si beaucoup se sont laïcisées depuis leurs origines. Ces références idéologiques différentes expliquent également des formes de bénévolat contrastées, plus caritatif et religieux aux EtatsUnis, plus militant et laïc ou tourné vers des activités d'expression et de sociabilité en France (Barthelemy, 2000).

Cette interprétation des caractéristiques des SSBL des deux pays par leur encastrement historique et institutionnel permet-elle également de rendre compte de leur évolution récente au cours de la dernière décennie ? C’est ce que l'on examinera maintenant à partir de données empiriques plus hétérogènes et moins comparables que celles précédemment utilisées.

\section{II - L'EVOLUTION RECENTE DES TIERS SECTEURS AMERICAIN ET FRANÇAIS ET LEUR REACTION FACE A LA CRISE}

Dans un premier point, on analysera l'évolution des tiers secteurs au cours de la période 1995-2007. Cette partie repose sur des données empiriques variées : deux enquêtes auprès des associations en 1999 et 2005 (Tchernonog, 2007) et le compte satellite des ISBL (Archambault et Kaminski, 2009) pour la France ; The Nonprofit sector Almanac et les premiers résultats de la phase 3 du programme Johns Hopkins pour les Etats-Unis. Comme ces données ne sont pas comparables avec celles de la première partie, on indiquera le sens des évolutions sans la plupart du temps les chiffrer. Dans un second temps on tentera de schématiser l'impact sur les ISBL de la crise financière, économique et sociale qui a frappé les deux pays à partir de 2008. Bien sûr, les données quantitatives ne sont pas disponibles au moment où cet article est rédigé et ce second point s'appuiera essentiellement sur des enquêtes qualitatives auprès des organisations et des dires d'experts. 


\section{a-L'évolution des ISBL au cours de la période 1995-2007 en France et aux Etats-Unis}

Certaines tendances sont communes aux deux pays : dans les deux pays, la période 1995-2007 est une période de croissance économique avec accroissement des inégalités de revenus, mais cette croissance a été plus rapide et plus inégalitaire aux Etats-Unis qu'en France, où l'innovation a été moins rapide et où un ample système de protection sociale a réduit les inégalités. On observe également une croissance rapide du nombre d'organisations nouvelles enregistrées ou déclarées chaque année dans les deux pays. Aux Etats-Unis, le nombre des ISBL enregistrées a augmenté entre 1995 et 2005 de 23\%, leurs ressources de $54 \%$ et leurs actifs de $77 \%$ alors que le produit intérieur brut ne croissait que de 35\% (Nonprofit Almanac, 2009) ${ }^{12}$. De même, on constate une croissance plus rapide de l'emploi dans le Tiers secteur que dans le reste de l'économie. Ainsi, entre 1993 et 2002, l'emploi associatif en France a progressé de 38\% quand l'emploi salarié dans les entreprises et la fonction publique ne progressaient que de $16 \%$ et $17 \%$ (Tchernonog, 2007). Une évolution comparable mais moins spectaculaire est observée aux Etats-Unis. Il en résulte que la part du SSBL dans l'emploi total passe de 6,3\% en 1997 à 8,1\% en 2007 aux Etats-Unis et de 3,7\% à 7,4\% en France ${ }^{13}$. Quelle que soient les réserves méthodologiques, il y a un effet de rattrapage indéniable en France.

Au cours de la période, on observe dans les deux pays une externalisation des services de santé et des services sociaux de l'Etat vers les ISBL, sur appel d'offres en compétition avec les entreprises lucratives. Le Tiers secteur se professionnalise des deux côtés de l'Atlantique sans que l'offre de travail bénévole se réduise, bien au contraire.

La progression du nombre et du pourcentage des bénévoles dans la population adulte est constante en France depuis 1990. Après avoir constamment augmenté de 1996 à 2005, le nombre de bénévoles et le taux de bénévolat déclinent aux Etats-Unis en 2006 et 200714.

Dans les deux pays, le nombre d'heures consacrées au bénévolat est extrêmement dispersé autour d'une moyenne annuelle de 99 heures en France et de 138 aux Etats-Unis ${ }^{15}$. Les caractéristiques sociodémographiques des bénévoles sont très proches : le bénévolat croît fortement avec le niveau d'études et le revenu; le taux de 
bénévolat est plus élevé pour les personnes en emploi que pour les inactifs ou les chômeurs; il crôt avec l'âge, atteint son maximum entre 35 et 55 ans puis décroît. En revanche les femmes sont plus souvent bénévoles que les hommes aux Etats-Unis, alors que c'est l'inverse en France, car l'orientation du bénévolat est différente. Près de la moitié du temps de travail bénévole, en effet, va aux ISBL du secteur «Culture, sports et loisirs » en France, où les hommes sont majoritaires, puis loin derrière à l'action sociale, alors qu'aux EtatsUnis plus du tiers de ce temps s'oriente vers les organisations religieuses, très féminisées, puis les institutions d'éducation et le développement communautaire.

Mais d'autres évolutions renforcent la spécificité des Tiers secteurs de chaque pays et peuvent être lus à travers le prisme de la théorie évolutionniste (Nelson et Winter, 1983). Selon cette théorie, les routines acquises et cristallisées dans les institutions, jointes à la rationalité limitée des acteurs, expliquent cette tendance à l'inertie des caractéristiques structurelles, souvent nommée «dépendance de sentier $»$.

Ainsi, la prédominance de la santé, $59 \%$ des ressources de l'ensemble des reporting charities en 2005 (Nonprofit Almanac, 2008), se renforce aux Etats-Unis alors que celle des services sociaux augmente encore en France. De même, la part relative des ressources marchandes des ISBL (ventes, participation des usagers au service rendu et revenus de placements) augmente aux Etats-Unis, atteignant $58 \%$ en $2005^{16}$. Cette évolution prolonge une tendance longue au désengagement du secteur public et à un recours accru au marché commencée sous la Présidence Reagan. Inversement, en France, les partenariats avec les pouvoirs publics se multiplient et la part du financement public dans les ressources du SSBL augmente légèrement puis stagne en fin de période, tout en se redéployant de l'Etat vers les collectivités territoriales (Tchernonog, 2007).

Un autre exemple de dépendance de sentier concerne la stabilité des comportements des donateurs. Au cours de la décennie observée, les incitations fiscales au don sont demeurées inchangées aux EtatsUnis alors qu'elles ont été fortement augmentées en France ${ }^{17}$. Cependant, le pourcentage de donateurs et le montant des dons a augmenté plus rapidement aux Etats-Unis qu'en France, en dépit d'incitations au don qui sont actuellement parmi les plus généreuses 
du monde : l'augmentation des dons en France ne correspondrait qu'à celle de la dépense fiscale, le «reste à charge» des donateurs demeurant inchangé. Cette moindre propension à donner des Français par rapport aux Américains s'explique évidemment par une conception différente de l'Etat qui détient dans la tradition jacobine le monopole de l'intérêt général (Rosanvallon, 2004).

Corrélativement, les fondations, déjà nombreuses aux Etats-Unis, se sont multipliées au cours de cette décennie de croissance inégalitaire, où les nouveaux philanthropes nés de la révolution numérique ont créé leur propre fondation (Bill et Melinda Gates, Hewlett Packard ou Google, par exemple). Leurs actifs ont considérablement augmenté ainsi que les subventions qu'elles versent aux autres ISBL. En France, les fondations créées par des particuliers restent peu nombreuses, même si elles augmentent à partir de la loi Aillagon de 2003., Les fondations d'entreprises en effet se multiplient depuis cette date, de même que les nouvelles formes de fondations (de coopération scientifique, universitaires, partenariales) initiées par cette loi, ainsi que les fonds de dotation créés en 2008.

La dépendance de sentier se retrouve aussi sur le plan idéologique : la Présidence W. Bush a été très favorable grâce au système des vouchers aux ISBL à référence religieuse (faith-based), notamment à la mouvance protestante évangélique, que ce soit dans le domaine scolaire ou celui de la santé. Dans le même temps, les ISBL françaises sont de plus en plus laïques et même celles qui se rattachent à une tradition charitable religieuse gomment cet héritage dans leurs messages adressés à une société française qui attache de moins en moins de prix aux valeurs religieuses. En revanche, les partenariats avec l'Etat ou les collectivités territoriales deviennent de plus en plus officiels, formalisés et durables dans des chartes, des conventions pluriannuelles d'objectifs, des référentiels élaborés en commun, des conférences de la vie associative. La nouvelle philanthropie introduit aux Etats-Unis la culture de l'évaluation et du résultat qui est celle des grandes entreprises capitalistes et la venture philanthropy réclame des ISBL davantage d'efficacité managériale, plus de transparence et une meilleure gouvernance, alors que leurs homologues cultivent leur différence en France. Enfin, les liens entre les associations et fondations et les coopératives ou mutuelles se consolident en France, fondés sur leur opposition à l'entreprise 
capitaliste, alors que le concept d'économie sociale n'existe pas aux Etats-Unis.

Ce renforcement des spécificités de chaque pays a des incidences sur l'impact de chaque SSBL sur la société. La marchandisation des ressources des ISBL aux Etats-Unis accentue leur orientation vers les classes moyenne et supérieure alors que beaucoup d'associations créées en France au cours de la période aident matériellement ou défendent les exclus et les sans-droits, avec ou sans financement public.

b-Quelle résistance des institutions sans but lucratif face à la crise financière, économique et sociale depuis 2008 ?

La crise a évidemment démarré aux Etats-Unis et ses effets destructeurs d'emplois se sont étalés sur les années 2008 et 2009. La France a suivi avec six mois de retard. Les deux pays ont mis en œuvre un plan de relance, beaucoup plus ample en valeur absolue et relative aux Etats-Unis qu'en France. Le plan de relance des EtatsUnis commence à porter ses fruits et les signes de redémarrage de la croissance depuis le début de 2010 y sont plus nets qu'en France. Quel a été l'impact de cette crise financière, économique et sociale sur les ISBL de chaque pays?

Là aussi, il y a des traits communs aux deux pays : la crise a créé de nouvelles formes de pauvreté liées à des suppressions d'emploi jamais connues à cette échelle depuis la Seconde Guerre mondiale, à de nouvelles vagues de sans domicile, par expulsion ou abandon de maisons impayées. Des deux côtés de l'Atlantique, ces nouveaux pauvres allongent les files d'attente devant les soup kitchens ou les Restos du Cœur. Les ISBL ont alors à faire face à un effet de ciseau : répondre à ces nouveaux besoins sociaux avec des ressources décroissantes, car la marchandisation accrue n'est pas une solution pour ces populations insolvables. Par ailleurs, la compétition devient plus dure entre ISBL pour obtenir des dons, avec un coût croissant et un rendement décroissant du fundraising. Dans le premier temps de la crise, les subventions publiques ou les remboursements pour les services sociaux ou de santé ont été retardés en France ou supprimés aux Etats-Unis (Aviv, 2009), provoquant des difficultés de trésorerie au mieux, et au pire le dépôt de bilan et le licenciement des salariés, comme c'est le cas de quelques associations de services à la personne en France. Certaines ISBL, plus nombreuses aux Etats-Unis qu'en 
France, fusionnent avec d'autres œuvrant dans le même secteur d'activité, mutualisent une partie de leurs moyens en infrastructure, en équipement ou en ressources humaines, gèlent les salaires et réduisent les frais généraux.

Inversement les différences sont marquées : il y a toujours moins de 2000 fondations en France (même si elles se multiplient depuis la loi Aillagon de 2003) et les actifs de ces fondations sont sans commune mesure avec ceux de leurs homologues américains; par ailleurs, les associations détiennent peu d'actifs financiers car, à part les associations reconnues d'utilité publique - moins de 2000 - elles n'ont pas le droit de posséder un patrimoine de rapport. Les ISBL françaises ont donc échappé largement à la crise financière et à l'effondrement des valeurs boursières qui a frappé les actifs des grandes fondations et public charities outre-Atlantique: la perte de valeur des actifs moyenne y est de 25 à $30 \%$ en 2009 , ce qui oblige la plupart des fondations à être plus sélectives dans le choix des projets qu'elles financent et les autres ISBL à se restructurer, comprimer leurs coûts et réduire ou supprimer certains programmes ${ }^{18}$.

Cependant, le plan de relance de l'administration Obama provoque en 2010 un rééquilibrage financier pour de nombreuses ISBL. En effet, des centaines de milliards de dollars ont été ou seront versées par l'Etat fédéral aux Etats fédérés et ceux-ci ont largement externalisé un grand nombre de prestations de services vers les ISBL des secteurs éducatif, sanitaire et social tout en assurant leur financement. Cette tendance très récente rapproche évidemment le tiers secteur américain du modèle européen de partenariat de bienêtre. Par ailleurs, les bénévoles, un peu moins présents en 2007 et 2008, reviennent plus nombreux et plus divers, après la grande mobilisation de la jeunesse que fut la campagne présidentielle d'Obama.

Pour les ISBL françaises en revanche, le plus dur reste sans doute à venir: les financements en provenance de l'Etat ont commencé à baisser et ne sont plus compensés par les collectivités territoriales, elles aussi en déficit. Les dons se maintiennent mais ne progressent pas et un effort de restructuration massif sera sans doute le lot des associations françaises, trop petites et trop nombreuses, dans les années à venir. Le caractère très contracyclique des ISBL et le décalage de la France par rapport à la crise et à la reprise américaine 
expliquent sans doute que l'emploi y augmente encore pendant les deux premiers trimestres de 2009, alors qu'il se contracte fortement dans le reste de l'économie.

\section{CONCLUSION}

On pourrait peut-être dire que le Tiers secteur américain est plus réactif et plus résilient que son homologue français. Plus proche par son statut juridique et la structure de ses ressources des entreprises commerciales, plus concentré et plus professionnalisé, plus orienté vers les classes moyennes, à l'exception de sa frange "compassionnelle», il dispose de fonds propres beaucoup plus importants, il est plus ouvert à l'innovation technologique et son comportement est plus procyclique. Le SSBL français est plus dispersé, plus effervescent, plus laïc et moins traditionnel, plus contracyclique de par sa forte imbrication dans les politiques de l'emploi. Il détecte les nouveaux besoins sociaux et il est à la source de nombreuses innovations sociales que l'Etat peut reprendre à son compte. Il revendique d'ailleurs un rôle de co-construction de l'intérêt général, en résistance aux résurgences jacobines, alors qu'aux EtatsUnis le Tiers secteur est par construction porteur de public good. Le SSBL français n'est donc pas simplement en retard conjoncturel ou structurel sur celui des Etats-Unis, il est héritier d'une histoire compliquée et de traditions différentes.

Dans quelle mesure la réforme du système de santé aux Etats-Unis va-t-elle orienter son tiers secteur vers une forme plus partenariale et vers les populations pauvres laborieuses? Quelles solutions seront trouvées en France au retrait progressif sans doute inéluctable du financement public? C'est le défi actuel qu'ont à relever les associations, expression fondamentale des sociétés démocratiques. «Pour que les hommes restent civilisés ou le deviennent, il faut que parmi eux l'art de s'associer se développe et se perfectionne dans le même rapport que l'égalité des conditions s'accroît», disait Tocqueville (Tocqueville, 1840). 
[1] Tocqueville publie De la Démocratie en Amérique (1835-1840) alors que la loi Guizot de 1834 interdit pratiquement la liberté de s'associer.

[2] On peut même dire que la liberté d'association n'a été complète qu'après 1981, date à laquelle a été supprimée l'autorisation administrative à laquelle étaient soumises les associations étrangères en France. Pour une histoire moins sommaire du secteur sans but lucratif en France, voir Archambault (1996 et 2001).

[3] SCN93, 4. 54

[4] ONU 2006 2.14. Ces cinq critères, qui doivent être simultanément réalisés, sont précisés dans les paragraphes 2.15 à 2.20 .

[5] En France, 92\% des ISBL sont des associations et 3\% des fondations. Le reste, ce sont les cultes et congrégations religieuses, les partis politiques, les syndicats, les comités d'entreprise et les organismes paritaires gérant des régimes de protection sociale facultatifs. Inversement, certaines associations ne sont pas des ISBL : celles qui sont lucratives (les cercles de jeu, par exemple), celles qui sont à la fois financées et contrôlées par la puissance publique (AFPA) ou par une entreprise, celles qui gèrent des régimes de protection sociale obligatoire (AGIRC, ARRCO) ou encore les organisations professionnelles ou patronales à adhésion obligatoire.

[6] Les public charities et les fondations sont classées en 501 (c) 3 : outre l'exemption fiscale, elles ont droit à la déductibilité fiscale des dons et legs qui leur sont adressés. Les autres organisations non lucratives, classées en 501 (c) 4 à 6 ont droit à l'exemption des impôts sur les sociétés, mais pas à la déductibilité fiscale des dons.

[7] Ce programme de comparaison internationale, à l'initiative de l'Université Johns Hopkins a concerné 13 pays dans sa première phase (1990-1995) et 37 pays dans sa phase 2 (1996-2002). Une méthodologie élaborée en commun a été utilisée par tous les pays comparés.

[8] La méthodologie élaborée collectivement par les 13 pays (dont la France) associés à la première phase du Programme Johns Hopkins a été validée par l'ONU et rendue compatible avec le système international de comptabilité nationale SCN93 dans le Manuel précité (ONU 2006).

[9] Ce chiffre a été recalculé en prenant pour base le temps de travail bénévole moyen déclaré à l'enquête "Conditions de vie, supplément vie associative» (INSEE, 2002) car les chiffres précédemment publiés reposaient sur une enquête qui surévaluait le temps de travail. En revanche le pourcentage de bénévoles est cohérent avec l'enquête INSEE. Cette erreur souligne l'importance d'une méthodologie standardisée pour la mesure du bénévolat (Archambault et Prouteau, 2009). 
[10] Tableau A - Structure du SSBL en France et aux Etats-Unis, 1995 (en pourcentage des salariés ETP du SSBL)

\begin{tabular}{|c|c|c|}
\hline Domaine d'activité selon ICNPO & $\begin{array}{c}\text { France } \\
\% \\
\end{array}$ & $\begin{array}{c}\text { Etats-Unis } \\
\% \\
\end{array}$ \\
\hline Culture, sports et loisirs & 12,1 & 7,3 \\
\hline Education et recherche & 20,7 & 21,5 \\
\hline Santé & 15,5 & 46,3 \\
\hline Services sociaux & 39,7 & 13,5 \\
\hline Environnement & 1,0 & 0,0 \\
\hline Développement local, logement & 5,5 & 6,3 \\
\hline Défense des droits et intérêts & 1,9 & 1,8 \\
\hline Fondations redistributrices & 0,0 & 0,3 \\
\hline Aide internationale & 1,8 & 0,0 \\
\hline Organisations professionnelles & 1,8 & 2,9 \\
\hline Total & 100 & 100 \\
\hline
\end{tabular}

Source : Salamon and associates, 1999.

[11] Selon ce principe, l'Etat n'intervient pas directement pour satisfaire un besoin social si une collectivité plus proche des personnes concernées, Etat fédéré, commune, association, a pu le faire. En revanche, il finance au moins partiellement la production de services par ces collectivités de proximité.

[12] On ne dispose malheureusement pas de données aussi précises et officielles en France actuellement. Cependant la tendance est la même.

[13] Cette forte progression en France est pour une part illusoire, car le premier chiffre est issu de la comparaison Johns Hopkins 2005, alors que le second provient des données INSEE Economie sociale, Associations, emploi en ETP, 2007 qui cernent des ensembles assez différents.

[14] Tableau B - Evolution du bénévolat dans les ISBL en France et aux Etats-Unis

\begin{tabular}{|c|c|c|c|c|c|c|}
\hline & 1990 & 1993 & 1996 & 2002 & 2005 & 2007 \\
\hline $\begin{array}{l}\text { France : Nombre } \\
\text { de bénévoles, en } \\
\text { millions }\end{array}$ & 7,9 & 9,0 & 10,4 & 12 & 14,2 & \\
\hline Taux de bénévolat & 19 & 20,8 & 23,4 & 26 & - & \\
\hline $\begin{array}{ll}\text { Etats-Unis : } & \\
\text { Nombre } & \text { de } \\
\text { bénévoles, } & \text { en } \\
\text { millions } & \\
\end{array}$ & & & & & 65,3 & 60,8 \\
\hline Taux de bénévolat & & & 22 & 26,7 & 28,8 & 26,2 \\
\hline
\end{tabular}

Sources: France: Enquêtes LES/ISL/Fondation de France pour 1990,1993, 1996 ; INSEE 2002 ; Tchernonog 2007 ; Etats-Unis : Current Population Survey, 2003, Bureau of Labor Statistics, 2009. 
[15] La médiane est de 50 heures aux Etats-Unis et sans doute plus basse en France. Cet écart entre moyenne et médiane traduit la forte asymétrie de la distribution des bénévoles. En France les deux tiers des bénévoles sont occasionnels et un tiers réguliers, parmi lesquels on trouve les plus fortes durées de travail. La proportion de bénévoles réguliers est nettement plus forte aux Etats-Unis.

[16] En 2005, la part du financement public est de 29\% (20\% de commandes publiques ou remboursements, liés essentiellement aux programmes Medicare et Medicaid) et celle des dons privés est de $12 \%$.

[17] Aux Etats-Unis les dons aux public charities et aux fondations sont intégralement déductibles du revenu imposable. L'avantage fiscal maximal est donc de $38 \%$, tranche supérieure de l'impôt progressif et les déductions sont plafonnées à 50\% du revenu imposable. En France, 50\% des dons aux ISBL en 1996, puis 60\% en 2003 et $66 \%$ en 2006 sont déductibles de l'impôt lui-même, avec un plafonnement qui est passé de $1,25 \%$ en 1996 à 20\% du revenu imposable en 2003.

[18] Une enquête récente auprès de 363 ISBL américaines montre que $83 \%$ d'entre elles ont connu des difficultés financières au cours des derniers mois, dont la moitié ont été estimées graves ou très graves. $51 \%$ ont vu leurs ressources diminuer et parmi elles, ce sont les dons des particuliers, des entreprises et des fondations qui ont le plus baissé. Les ISBL qui ont le plus souffert sont les théâtres et orchestres, très tributaires aux EtatsUnis des dons ; celles qui ont connu le moins de difficultés financières sont les écoles et les universités. (Salamon et alii, 2009)

\section{BIBLIOGRAPHIE}

Archambault E., 1996, Le secteur sans but lucratif. Associations et Fondations en France, Paris, Economica.

Archambault E, 2001, « Historical Roots of the Nonprofit sector in France » Nonprofit and Voluntary Sector Quarterly, vol. 30, n² 2, p. 204-221.

Archambault E, (2009), "The Third Sector in Europe : Does it Exhibit a Converging Movement» in B. Enjolras and K.H. Sivesind Edrs, Civil Society in Comparative Perspective, Comparative Social Research, vol. 26, p. 3-24.

Archambault E. et P. Kaminski, 2009, «La longue marche vers un compte satellite de l'économie sociale » Annals of Public and cooperative economy, vol. $80, \mathrm{n}^{\circ} 2$, p. $225-246$.

Archambault E. et Prouteau L., 2009, «Mesurer le bénévolat pour en améliorer la connaissance et répondre à une recommandation internationale » RECMA Revue internationale de l'économie sociale, vol. 88, $\mathrm{n}^{\circ}$ 314, octobre, p.84-104.

Aviv D, 2009, Conférence invitée au Colloque du Comité de la Charte, Paris, 8 octobre.

Barthelemy M., 2000, Associations : un nouvel âge de la participation? Paris, Presses de Sciences-Po.

Bureau of Labor Statistics (2009), Volunteering in the United States. 
CEVIPOF, 2010, Les associations devant la démocratie : France/Etats-Unis, Paris, Journée d'études du 15 janvier 2010, notamment interventions de M. Barthelemy, L. Jaume, O. Zunz.

Defourny J. et Nyssens M., 2008, "Conceptions of Social Enterprise in Europe and the United States: Convergences and divergences » EMES Summer School, Corte.

Esping-Andersen G., 1990, The Three Worlds of Welfare Capitalism. Princeton, Princeton University Press.

Nelson R. et Winter S.,1982, An evolutionary theory of economic change, Harvard University Press.

National Center for charitable statistics, 2009, Nonprofit Almanac Public Charities, giving and volunteering, Urban Institute.

North D., 1990, Institutions, Institutional change and Economic Performance, Cambridge, Cambridge University Press.

Rosanvallon P., 2004, Le modèle politique français. La société française contre le jacobinisme de 1789 à nos jours, Paris, Le Seuil.

Salamon L. et Anheier H., 1998, "Social origins of civil society ", Voluntas, vol. $9, \mathrm{n}^{\circ} 3$, p. $213-248$.

Salamon L. et Sokolowski W., 2004, Global Civil Society. Dimensions of the Nonprofit Sector, volume 2, Baltimore, The Johns Hopkins Center for Civil Society Studies and Kumarian Press.

Salamon L., Geller S. et Spence K., 2009, Impact of the 2007-2009 Economic Recession on Nonprofit Organizations, Baltimore, Johns Hopkins University, Listening Post Project.

System of National Accounts, 1993, United Nations Publications.

Tchernonog V., 2007, Le paysage associatif français, Mesures et évolutions, Juris Associations, Dalloz.

Tocqueville A. de, 1835 et 1840, De la Démocratie en Amérique, Paris, Gallimard, 1951.

United Nations Statistics Division, 2003, Handbook on Non-Profit Institutions in the System of National Accounts, Statistical Papers, Series F, $\mathrm{n}^{\circ} 91$ (traduction française parue en 2006).

\section{RESUME}

La comparaison des secteurs non lucratifs américain et français commence par un état des lieux en 1995. Les données empiriques strictement comparables sont interprétées par les ideal-types libéral anglo-saxon et partenarial européen de bien-être. Dans un deuxième temps, on analyse l'évolution depuis ce point de départ jusqu'à 2007, en insistant sur le renforcement des spécificités de chaque pays, même si certaines tendances se retrouvent en France comme aux Etats-Unis. Enfin on essaie de voir l'impact de la crise financière, économique et sociale sur les deux Tiers secteurs et leur résilience respective. 